\title{
THE METHOD OF DUAL INTEGRAL EQUATIONS FOR ANALYSIS OF HEAT TRANSFER PROCESSES
}

\author{
P. A. MANDRIK \\ Belarusian State University \\ Fr. Scoriny av. 4, 220050, Minsk, Belarus \\ E-mail: mandrik@fpm.bsu.unibel.by
}

Received September 17, 2001; revised November 20, 2001

\begin{abstract}
The paper is devoted to the study of a heat conduction equations with mixed boundary conditions on a surface of a researched solid. The method based on the Laplace and Hankel transforms is suggested for the first time to reduce such differential problems to dual integral equations and to obtain the solution in the close form. Method allows to determine analytically regularities of time-space development of appropriate temperature fields.
\end{abstract}

\section{PROBLEM FORMULATION}

We consider the heat transfer process where the heat flows to an isotropic half-space solid through a circle of a known radius on the border surface. We note that the solution of similar problems of mathematical physics is also possible for other solids and regions (see, for example, [2], [3], [6], [8]-[11]).

The mathematical model based on the equation

$$
\theta_{r r}(r, z, \tau)+\frac{1}{r} \theta_{r}(r, z, \tau)+\theta_{z z}(r, z, \tau)=\frac{1}{a} \theta_{\tau}(r, z, \tau) \quad(r>0, z>0, \tau>0)
$$

with initial condition

$$
\theta(r, z, 0)=0 \quad(r \geq 0, z \geq 0)
$$

symmetry condition 


$$
\theta_{r}(0, z, \tau)=0 \quad(z \geq 0, \tau \geq 0)
$$

and conditions at infinity

$$
\theta_{r}(\infty, z, \tau)=\theta_{z}(r, \infty, \tau)=0 \quad(r \geq 0, z \geq 0, \tau \geq 0) .
$$

Here $\theta(r, z, \tau)=T(r, z, \tau)-T_{0} ; r \geq 0$ and $z \geq 0$ are a cylindrical co-ordinates; $\tau \geq 0$ is a time co-ordinate; $T(r, z, \tau)$ is a current temperature of half-space; $T_{0}=$ const is an initial temperature; $a>0$ is a temperature conductivity factor.

On a surface $z=0$, generally speaking, the mixed boundary conditions of the third kind can be given in the form [7]

$$
\begin{gathered}
\alpha_{1} \theta(r, 0, \tau)-\lambda \theta_{z}(r, 0, \tau)=\alpha_{1}\left(T_{1 c}(r, \tau)-T_{0}\right)+q_{1}(r, \tau), 0<r<R, \\
\alpha_{2} \theta(r, 0, \tau)-\lambda \theta_{z}(r, 0, \tau)=\alpha_{2}\left(T_{2 c}(r, \tau)-T_{0}\right)+q_{2}(r, \tau), R<r<\infty .
\end{gathered}
$$

Here $\lambda>0$ is a heat conductivity factor, $\alpha_{1}$ and $\alpha_{2}$ are a heat transfer factors on a surface $z=0$ with mediums having nonstationary temperatures $T_{1 c}(r, \tau)$ and $T_{2 c}(r, \tau)$ in the corresponding ranges of a modification of cylindrical coordinate $r, q_{1}(r, \tau)$ and $q_{2}(r, \tau)$ are the corresponding densities of surface heat sources on $z=0$ inside of the circle $(r<R)$ and outside of the circle $(r>R)$.

\section{INTEGRAL TRANSFORMS}

To solve the problem (1.1)-(1.6) we shall apply Laplace and Hankel integral transforms

$$
\begin{gathered}
\bar{\theta}(r, z, s)=\int_{0}^{\infty} \theta(r, z, \tau) \exp (-s \tau) d \tau \quad(\operatorname{Re} s>0), \\
\bar{\theta}_{H}(p, z, s)=\int_{0}^{\infty} \int_{0}^{\infty} \theta(r, z, \tau) \exp (-s \tau) J_{0}(p r) r d r d \tau \quad(\operatorname{Re} s>0),
\end{gathered}
$$

where $J_{0}(p r)$ is the Bessel function of the first kind (see [15]).

Next we shall assume $\operatorname{Re} s>0$ by default every time, when a complex $L$-parameter $s$ will appear.

Applying (2.2) to the equation (1.1), taking (1.2)-(1.4) into account and using an inversion formula for the Hankel transform, it is directly verified that

$$
\bar{\theta}_{H}(p, z, s)=\frac{1}{p} \bar{A}(p, s) \exp \left(-z \sqrt{p^{2}+\frac{s}{a}}\right),
$$




$$
\bar{\theta}(r, z, s)=\int_{0}^{\infty} \bar{A}(p, s) \exp \left(-z \sqrt{p^{2}+\frac{s}{a}}\right) J_{0}(p r) d p,
$$

where $\bar{A}(p, s)$ is an auxiliary analytical image-function.

Taking the solution (2.4) into account at $z=0$ and using the Laplace transform to mixed boundary conditions (1.5) and (1.6) we come to the dual integral equations with $L$-parameter

$$
\begin{aligned}
& \int_{0}^{\infty}\left(\alpha_{1}+\lambda \sqrt{p^{2}+\frac{s}{a}}\right) \bar{A}(p, s) J_{0}(p r) d p \\
& =\alpha_{1}\left(\bar{T}_{1 c}(r, s)-\frac{T_{0}}{s}\right)+\bar{q}_{1}(r, s), \quad 0<r<R, \\
& \int_{0}^{\infty}\left(\alpha_{2}+\lambda \sqrt{p^{2}+\frac{s}{a}}\right) \bar{A}(p, s) J_{0}(p r) d p \\
& =\alpha_{2}\left(\bar{T}_{2 c}(r, s)-\frac{T_{0}}{s}\right)+\bar{q}_{2}(r, s), \quad R<r<\infty
\end{aligned}
$$

From here we have to define an analytical image-function $\bar{A}(p, s)$.

Note, that if $s \rightarrow 0$ (that corresponds $\tau \rightarrow \infty$ ), then from the equations (2.5) and (2.6) we come to dual stationary integral equations which were investigated for the Laplace equation with mixed boundary conditions (see, for example, [13], [14]).

We shall consider only the cases of mixed discontinuous boundary conditions (1.5) and (1.6), which can be reduced to the appropriate dual integral equations with $L$-parameter. In the other cases we do not need the application of a method of dual integral equations, because we have the nonstationary Dirichlet problem (for $\alpha_{1} \rightarrow \infty$ and $\alpha_{2} \rightarrow \infty$ ) and nonstationary Neumann problem (for $\alpha_{1}=\alpha_{2}=0$ ) in an isotropic half-space with unmixed discontinuous boundary conditions of the first and second kinds. Those problems are directly solved by using the inversion formulas of Laplace and Hankel transforms.

When $\alpha_{1} \rightarrow \infty$ and $\alpha_{2} \geq 0$ the function of excess image-temperature $\bar{T}_{1 c}(r, s)-T_{0} / s$ is given on a surface $z=0$ inside the circle $0<r<R$ while outside of this circle, the surface source $\bar{q}_{2}(r, s)$ is exchanged by a heat with a medium having image-temperature $\bar{T}_{2 c}(r, s)$ and heat transfer factor $\alpha_{2}$. In this case the dual integral equations (2.5) and (2.6) are transforming to the form

$$
\int_{0}^{\infty}\left[1-\bar{g}_{1}(p, s)\right] \bar{C}_{1}(p, s) J_{0}(p r) d p=\bar{T}_{1 c}(r, s)-\frac{T_{0}}{s}, \quad 0<r<R,
$$




$$
\int_{0}^{\infty} \bar{C}_{1}(p, s) \sqrt{p^{2}+\frac{s}{a}} J_{0}(p r) d p=\frac{1}{\lambda} \bar{f}_{2}(r, s), \quad R<r<\infty,
$$

where

$$
\begin{gathered}
\bar{C}_{1}(p, s)=\left[1+\frac{\alpha_{2}}{\lambda \sqrt{p^{2}+\frac{s}{a}}}\right] \bar{A}(p, s), \quad \bar{g}_{1}(p, s)=\frac{\alpha_{2}}{\alpha_{2}+\lambda \sqrt{p^{2}+\frac{s}{a}}}, \\
\bar{f}_{2}(r, s)=\alpha_{2}\left(\bar{T}_{2 c}(r, s)-\frac{T_{0}}{s}\right)+\bar{g}_{2}(r, s) .
\end{gathered}
$$

When $\alpha_{1} \geq 0$ and $\alpha_{2} \rightarrow \infty$ the surface source $\bar{q}_{1}(r, s)$ is exchanged by a heat with a medium having image-temperature $\bar{T}_{1 c}(r, s)$ and heat transfer factor $\alpha_{1}$ on a surface $z=0$ inside the circle $0<r<R$ while outside of the circle, the function of excess image-temperature $\bar{T}_{2 c}(r, s)-T_{0} / s$ is given. In this case the dual integral equations (2.5) and (2.6) are transforming to the other standard form

$$
\begin{gathered}
\int_{0}^{\infty}\left[1-\bar{g}_{2}(p, s)\right] \sqrt{p^{2}+\frac{s}{a}} \bar{C}_{2}(p, s) J_{0}(p r) d p=\frac{1}{\lambda} \bar{f}_{1}(r, s), \quad 0<r<R, \\
\int_{0}^{\infty} \bar{C}_{2}(p, s) J_{0}(p r) d p=\bar{T}_{2 c}(r, s)-\frac{T_{0}}{s}, \quad R<r<\infty
\end{gathered}
$$

where

$$
\begin{gathered}
\bar{C}_{2}(p, s)=\bar{A}(p, s) \quad \bar{g}_{2}(p, s)=-\frac{\alpha_{1}}{\lambda \sqrt{p^{2}+\frac{s}{a}}}, \\
\bar{f}_{1}(r, s)=\alpha_{1}\left(\bar{T}_{1 c}(r, s)-\frac{T_{0}}{s}\right)+\bar{q}_{1}(r, s) .
\end{gathered}
$$

In the general case when $\alpha_{1} \geq 0, \alpha_{2} \geq 0$, and $\alpha_{1}^{2}+\alpha_{2}^{2} \neq 0$ the substitution

$$
\bar{C}(p, s)=\left(\alpha_{2}+\lambda \sqrt{p^{2}+\frac{s}{a}}\right) \bar{A}(p, s)
$$

yields the dual integral equations with $L$-parameter

$$
\begin{gathered}
\int_{0}^{\infty}[1-\bar{g}(p, s)] \bar{C}(p, s) J_{0}(p r) d p=\bar{f}_{1}(r, s), \quad 0<r<R \\
\int_{0}^{\infty} \bar{C}(p, s) J_{0}(p r) d p=\bar{f}_{2}(r, s), \quad R<r<\infty
\end{gathered}
$$


where

$$
\bar{g}(p, s)=\frac{\alpha_{2}-\alpha_{1}}{\alpha_{2}+\lambda \sqrt{p^{2}+\frac{s}{a}}} .
$$

Thus, the solution of the mixed two-dimensional axially symmetric nonstationary heat conduction problems for a half-space with heat input through a circle of a known radius is reduced to the investigation of the standard dual integral equations with $L$-parameter (2.7)-(2.8) or (2.9)-(2.10), or (2.11)(2.12). It is required to define the corresponding unknown analytical imagefunctions $\bar{C}_{1}(p, s)$ or $\bar{C}_{2}(p, s)$, or $\bar{C}(p, s)$ in those equations.

\section{SOLUTION OF DUAL INTEGRAL EQUATION}

We consider the solution of the dual integral equations (2.7) and (2.8) in the case $\bar{f}_{2}(r, s)=0$ and $\bar{g}_{1}(p, s)=0$. This case is really general, because determining additional function $\bar{f}_{2}(r, s)$ by a zero on an interval $0<r<R$ and including known function $\bar{f}_{2}(r, s)$ to the right hand side of the equation (2.7), the equation (2.8) is reducing (see [12]) to the homogeneous one. In this case the dual integral equations (2.7) and (2.8) take the form

$$
\begin{gathered}
\int_{0}^{\infty} \bar{C}_{1}(p, s) J_{0}(p r) d p=\bar{T}_{1 c}(r, s)-\frac{T_{0}}{s}, \quad 0<r<R, \\
\int_{0}^{\infty} \bar{C}_{1}(p, s) \sqrt{p^{2}+\frac{s}{a}} J_{0}(p r) d p=0, \quad R<r<\infty .
\end{gathered}
$$

By analogy with Snedonn's and Uflend's methods (see [12], [13]) we shall introduce an unknown analytical function $\bar{\varphi}(r, s)$ connected with $\bar{C}_{1}(p, s)$ by the relation

$$
\bar{C}_{1}(p, s)=\frac{p}{\sqrt{p^{2}+\frac{s}{a}}} \int_{0}^{R} \bar{\varphi}(t, s) \cos \left(t \sqrt{p^{2}+\frac{s}{a}}\right) d t .
$$

The substitution of this relation into (3.2) ensures the validity of the relation (3.2) by the corresponding discontinuous integral at $R<r<\infty$ (see [4], [1, p. 175]). If we substitute (3.3) into the first dual equation (19) and then take some transformations, we come to the integral equation for the definition of unknown analytical image-function $\bar{\varphi}(r, s)$ :

$$
\begin{array}{r}
\int_{0}^{r} \frac{\operatorname{ch}\left(\sqrt{\frac{s}{a}\left(r^{2}-t^{2}\right)}\right)}{\sqrt{r^{2}-t^{2}}} \bar{\varphi}(t, s) d t-\int_{0}^{R} \frac{\sin \left(\sqrt{\frac{s}{a}\left(t^{2}-r^{2}\right)}\right)}{\sqrt{t^{2}-r^{2}}} \bar{\varphi}(t, s) d t \\
=\bar{T}_{1 c}(r, s)-\frac{T_{0}}{s}, \quad 0<r<R .
\end{array}
$$


To solve the equation (3.5) we take some transformations and reduce this equation to the form (see [1], [6])

$$
\bar{\varphi}(r, s)-\frac{1}{\pi} \int_{0}^{R} \bar{\varphi}(t, s) \bar{K}_{1}(r, t, s) d t=\bar{F}_{1}(r, s), \quad 0<r<R
$$

where

$$
\begin{gathered}
\bar{K}_{1}(r, t, s)=\frac{\sin \left((t-r) \sqrt{\frac{s}{a}}\right)}{t-r}+\frac{\sin \left((t+r) \sqrt{\frac{s}{a}}\right)}{t+r}, \\
\bar{F}_{1}(r, s)=\frac{2}{\pi} \frac{d}{d r} \int_{0}^{r} \frac{\left(\bar{T}_{1 c}(r, s)-\frac{T_{0}}{s}\right) \mu \cos \left(\sqrt{\frac{s}{a}\left(r^{2}-\mu^{2}\right)}\right)}{\sqrt{r^{2}-\mu^{2}}} d \mu .
\end{gathered}
$$

In the case $\bar{T}_{1 c}(r, s)=\frac{T_{c}}{s} \neq \frac{T_{0}}{s}$ the method of definition of unknown analytical function $\bar{\varphi}(r, s)$ from the integral equation with $L$-parameter (3.5) was developed in [5]. In this method the analytical function $\bar{\varphi}(t, s)$ is represented by the series

$$
\bar{\varphi}(t, s)=\frac{1}{s} \exp \left(-R \sqrt{\frac{s}{a}}\right) \sum_{n=0}^{\infty} \varphi_{n}(t)(\sqrt{s})^{n}
$$

and the analytical kernel $\bar{K}_{1}(r, t, s)$ is represented by the power series

$$
\bar{K}_{1}(r, t, s)=\sum_{m=0}^{\infty} C_{m}(t, r)(\sqrt{s})^{m}
$$

where

$$
C_{m}(t, r)=\frac{\sin \left(m \frac{\pi}{2}\right)}{m !}\left(\frac{1}{\sqrt{a}}\right)^{m}\left[(t+r)^{m-1}+(t-r)^{m-1}\right] .
$$

Then the equation (3.5) takes the form

$$
\begin{array}{r}
\pi \sum_{n=0}^{\infty} \varphi_{n}(r)(\sqrt{s})^{n}=2\left(T_{c}-T_{0}\right) \exp \left(R \sqrt{\frac{s}{a}}\right) \cos \left(r \sqrt{\frac{s}{a}}\right) \\
+\int_{0}^{R} \sum_{m=0}^{\infty} C_{m}(t, r)(\sqrt{s})^{m} \sum_{n=0}^{\infty} \varphi_{n}(t)(\sqrt{s})^{n} d t .
\end{array}
$$


From here (after some transformations) we obtain the equation for $\varphi_{n}(r)$ :

$$
\begin{aligned}
& \pi \sum_{n=0}^{\infty} \varphi_{n}(r)(\sqrt{s})^{n}=2\left(T_{c}-T_{0}\right) \sum_{n=0}^{\infty} D_{n}(R, r)(\sqrt{s})^{n} \\
& +\sum_{n=0}^{\infty} \sum_{m=0}^{n}(\sqrt{s})^{n} \int_{0}^{R} C_{m}(t, r) \varphi_{n-m}(t) d t, \quad 0<r<R,
\end{aligned}
$$

where

$$
D_{n}(R, r)=\frac{1}{n !}\left(\frac{R}{\sqrt{a}}\right)^{n} \sum_{j=0}^{n}\left(\begin{array}{c}
n \\
j
\end{array}\right)\left(\frac{r}{R}\right)^{j} \cos \left(j \frac{\pi}{2}\right), \quad\left(\begin{array}{c}
n \\
j
\end{array}\right)=\frac{n !}{j !(n-j) !} .
$$

It is obvious that $C_{0}(t, r)=0$ and the equation (3.9) is not an integral equation for the definition of $\varphi_{n}(r)$, and we obtain the recurrence formula for the sequential definition of functional factors $\varphi_{n}(r)$ :

$$
\varphi_{n}(r)=\frac{2}{\pi}\left(T_{c}-T_{0}\right) D_{n}(R, r)+\frac{1}{\pi} \sum_{m=0}^{n} \int_{0}^{R} C_{m}(t, r) \varphi_{n-m}(t) d t, \quad 0<r<R .
$$

So, the formula (3.9) yields

$$
\begin{gathered}
\varphi_{0}(r)=\frac{2}{\pi}\left(T_{c}-T_{0}\right), \quad \varphi_{1}(r)=\frac{2 R(2+\pi)}{\pi^{2} \sqrt{a}}\left(T_{c}-T_{0}\right), \\
\varphi_{2}(r)=\left(\frac{R^{2}-r^{2}}{\pi a}+\frac{4 R^{2}(2+\pi)}{\pi^{3} a}\right)\left(T_{c}-T_{0}\right),
\end{gathered}
$$

and so on.

Moreover, it is proved that in this case for functional factors $\varphi_{n}(r)$ the expression

$$
\varphi_{n}(r)=2\left(T_{c}-T_{0}\right)(\sqrt{a})^{-n} \sum_{i=0}^{n} \beta_{n, i} R^{n-i} r^{i}
$$

can be obtain. Here constant numerical coefficients $\beta_{n, i}$ can be define from the recurrent formula

$$
\begin{array}{r}
\beta_{n, i}=\frac{1}{\pi n !}\left(\begin{array}{c}
n \\
i
\end{array}\right) \cos \left(i \frac{\pi}{2}\right)+\left[1+(-1)^{i}\right] \sum_{j=i+1}^{n} \frac{1}{\pi j !}\left(\begin{array}{c}
j-1 \\
i
\end{array}\right) \sin \left(j \frac{\pi}{2}\right) \times \\
\times \sum_{k=0}^{n-j} \frac{1}{j-i+k} \beta_{n-j, k}, \quad i=0,1, \ldots, n-1 ;
\end{array}
$$




$$
\beta_{n, n}=\frac{1}{\pi n !} \cos \left(n \frac{\pi}{2}\right)
$$

Substituting expression for $\varphi_{n}(r)$ from (3.10) into (3.6) and taking the formulas (3.3) and (2.4) into account we obtain the formula for the solution

$$
\bar{\theta}(r, z, s)=\frac{2\left(T_{c}-T_{0}\right)}{s} \exp \left(-R \sqrt{\frac{s}{a}}\right) \sum_{n=0}^{\infty}\left(\sqrt{\frac{s}{a}}\right)^{n} \sum_{i=0}^{n} \beta_{n, i} R^{n-i} \bar{S}_{i}(r, z, s),
$$

where

$$
\bar{S}_{i}(r, z, s)=\int_{0}^{R} t^{i} d t \int_{0}^{\infty} \frac{p J_{0}(p r)}{\sqrt{p^{2}+\frac{s}{a}}} \exp \left(-z \sqrt{p^{2}+\frac{s}{a}}\right) \cos \left(t \sqrt{p^{2}+\frac{s}{a}}\right) d p .
$$

\section{REFERENCES}

[1] V.P. Kozlov and P.A. Mandrik. Systems of Integral and Differential Equations with L-parameter in Problems of Mathematical Physics and Identification Methods of Heat Characteristics. BGU Publ., Minsk. 2000 (in Russian)

[2] V.P. Kozlov and P.A. Mandrik. Nonstationary temperature fields into isotropic halfspace under mixed boundary conditions, reference to engineering processes of a laser medicine. Jour. Eng. Phys. and Thermophys., 73 (3), 2000, 637-644.(in Russian)

[3] V.P. Kozlov and P.A. Mandrik. The solution of nonlinear two-dimensional differential transfer equations with discontinuous boundary conditions. Jour. Eng. Phys. and Thermophys., 74 (2), 2001, 153-156.(in Russian)

[4] V.P. Kozlov, P.A. Mandrik and N.I. Yurchuk. The method of dual integral equations into L-image range for solution of two-dimensional nonstationary heat transfer problems under mixed boundary conditions. Jour. Eng. Phys. and Thermophys., 71 (4), 1998, 734-743.(in Russian)

[5] V.P. Kozlov, P.A. Mandrik and N.I. Yurchuk. The solution method of integral equations with nonsingular kernels into L-image region. Vestn. Belorus. Univer., Ser. 1 (2), 1999, 37-42.(in Russian)

[6] V.P. Kozlov, P.A. Mandrik and N.I. Yurchuk. The method of dual integral equations into Laplace transform range for solution of nonstationary heat transfer problems under mixed discontinuous boundary conditions. Jour. Eng. Phys. and Thermophys., 72 (3), 1999, 555-571.(in Russian)

[7] A.V. Lykov. Theory of Heat Transfer. Vysshaya shkola Publ., Moskva. (in Russian)

[8] P.A. Mandrik. The dual integral equations for half-space heating problem through infinitely long band. Vestn. Belorus. Univer., Ser. 1 (1), 2000, 75-76.(in Russian)

[9] P.A. Mandrik. The method of dual integral equations with L-parameter in nonstationary heat transfer problems with mixed boundary conditions for infinitely long band. Jour. Eng. Phys. and Thermophys., 73 (5), 2000, 902-906.(in Russian)

[10] P.A. Mandrik. The solution method of dual integral equations with trigonometric functions into Laplace transform range. Vestn. Belorus. Univer., Ser. 1 (1), 2001, 89-91.(in Russian)

[11] P.A. Mandrik. The solution of heat conduction equations with mixed boundary conditions on a surface of isotropic half-space. Differentsial'nye Uravneniya, 37 (2), 2001, 238-241.(in Russian) 
[12] I.N. Sneddon. Mixed Boundary Value Problems in Potential Theory. North Holland Publ., Amsterdam. 1966

[13] Ya.S. Uflend. Method of Dual Equations in Mathematical Physics Problems. Nauka Publ., Leningrad. 1977 (in Russian)

[14] G.N. Watson. Theory of Bessel Functions. Nauka Publ., Moskva. 1949 (in Russian)

[15] N.A. Virchenko. Dual (Triple) Integral Equations. Kiev University Publ., Kiev. 1989 (in Russian)

\section{Dualiuju integralinių lygčių metodas šilumos pernešimo procesų analizèje}

P.A. Mandrik

Straipsnyje nagrinèjamos šilumos pernešimo lygtys su mišriomis kraštinėmis sąlygomis tiriamo kūno paviršiuje. Metodas remiasi Laplaso ir Hankelio trasformacijomis, kurios suveda ši diferencialinị uždavinị į dualiąsias integralines lygtis. Metodas leidžia analitiškai apibrèžti ¿̇vairių temperatūrinių laukų laikinį - erdvinį kitimą. 